\title{
Percepções de estudantes de enfermagem acerca das dimensões estruturais da simulação clínica
}

\author{
Perceptions of nursing students on the structural dimensions of clinical simulation
}

Raphael Raniere de Oliveira Costa (D) ${ }^{1} \bowtie$, Soraya Maria de Medeiros ${ }^{(\mathbb{D})^{2}}$, José Carlos Amado Martins (in memoriam) (D) ${ }^{3}$, Verónica Rita Dias (D) ${ }^{3}$

1 Universidade Federal do Rio Grande do Norte, Escola Multicampi de Ciências Médicas do Rio Grande do Norte. Caicó, Rio Grande do Norte, Brasil.

2 Universidade Federal do Rio Grande do Norte, Departamento de Enfermagem. Natal, Rio Grande do Norte, Brasil.

Escola Superior de Enfermagem de Coimbra. Coimbra, Portugal.

Como citar este artigo (How to cite this article):

Costa RRO, Medeiros SM, Martins JCA, Dias VR. Percepções de estudantes de enfermagem acerca das dimensões estruturais da simulação clínica (Perceptions of nursing students on the structural dimensions of clinical simulation). Sci Med. 2019;29(1):e32972. https://doi.org/10.15448/1980-6108.2019.1.32972.

\section{RESUMO}

OBJETIVOS: Identificar a percepção de estudantes de enfermagem sobre as dimensões estruturais do design de cenários de simulação de alta fidelidade na imunização de adultos no contexto da atenção primária à saúde.

MÉTODOS: Estudo descritivo, exploratório, quantitativo desenvolvido em uma universidade pública federal brasileira no período de maio a junho de 2017. A amostra final foi de 17 estudantes. A Escala de Design da Simulação do tipo Likert de cinco itens foi utilizada para avaliar a estruturação do cenário de simulado.

RESULTADOS: A maioria dos estudantes são adultos jovens e do sexo feminino (82,4\%). Na análise de consistência interna, a Escala de Design de Simulação apresentou um alpha de Cronbach total de 0,930. Em todas as dimensões da escala, obteve-se médias globais iguais ou superiores a 4,60. As dimensões que apresentaram maior concordância e importância entre os estudantes foram, respectivamente, a resolução de problemas e o realismo.

CONCLUSÕES: Na percepção dos estudantes, os cenários simulados foram claros e objetivos em relação à proposta e à execução. Além disso, estes sentiram-se apoiados durante o processo de aprendizagem, o que permitiu explorar e resolver os problemas apresentados durante a simulação. O feedback fornecido foi relevante e oportunizou a reflexão dos estudantes quanto às suas práticas e posturas. Por fim, o realismo foi destacado como um aspecto importante pelos pesquisados.

DESCRITORES: Simulação; Ensino de Enfermagem; Atenção Primária à Saúde.

\section{ABSTRACT}

AIMS: To identify the perception of nursing students about the structural dimensions of the design of high-fidelity simulation scenarios in adult immunization scenarios in the context of primary health care.

METHODS: Descriptive, exploratory, quantitative study developed at a Brazilian federal public university from May to June 2017. The final sample consisted of 17 students. The 5-item Likert simulation design Scale was used to evaluate the design of simulation scenarios.

RESULTS: The majority of students are young and female adults (82.4\%). In the internal consistency analysis, the simulation design scale presented a total Cronbach's alpha of 0.930 . In all dimensions of the scale, overall averages were equal to or greater than 4.60. The dimensions that showed the greatest agreement and importance among students were, respectively, problem solving and realism.

CONCLUSIONS: In the students' perception, the simulated scenarios were clear and objective in relation to the proposal and the execution In addition, they felt supported during the learning process, which allowed to explore and solve the problems presented during the simulation The feedback provided was relevant and provided opportunities for students to reflect on their practices and attitudes. Finally, realism was highlighted as an important aspect by those surveyed.

KEYWORDS: Simulation; Nursing Education; Primary Health Care. 
Abreviatura: S.M.A.R.T, Specific, Measurable, Achievable, Realistic, Time phased.

\section{INTRODUÇÃO}

Para a National League for Nursing, a simulação é uma técnica que usa uma situação ou ambiente criado para permitir que as pessoas experimentem uma representação de um evento real com a finalidade de prática, aprendizado, avaliação, teste ou para obter entendimento de sistemas ou ações humanas [1].

Há evidências que a simulação favorece o desenvolvimento de habilidades psicomotoras, de habilidades sociais e de comunicação, da criatividade, do trabalho em equipe e colaboração, do pensamento crítico e do aprendizado clínico. Além disso, estudos referem a sua contribuição na busca de informações, no raciocínio, na resolução de problemas, na avaliação e na tomada de decisão [2].

Dada a sua aplicabilidade, o uso da simulação para a formação em enfermagem e em outras áreas da saúde continua a crescer e se desenvolver [3]. Além disso, vem ganhando aceitação por estudantes, docentes e órgão de acreditação em saúde [2].

Embora tenha apresentado uma expansão considerável nos últimos anos, algumas dificuldades se fazem presentes no desenvolvimento de estudos nesta área. A falta de financiamento, a falta de treinamento de simulação para docentes e outros colaboradores, e a falta de apoio das instituições que realizam pesquisas de simulação são alguns dos desafios da investigação nesta área [4].

É válido ressaltar que a estratégia da simulação possui diferentes níveis de fidelidades - baixa, média e alta - e pode utilizar diferentes materiais e métodos - simuladores, manequins, atores, entre outros. Cada nível de fidelidade e material utilizado pode apresentar diferentes resultados de aprendizado [1]. Neste estudo, destacam-se os conceitos de simulação de alta fidelidade e de paciente-padrão.

Simulação de alta fidelidade é um cenário de assistência que usa um paciente padronizado ou um simulador de corpo inteiro que pode ser programado para responder a mudanças afetivas e psicomotoras [5]. Paciente-padrão, são atores treinados para atuar e reproduzir comportamentos de usuários em diversas situações e estabelecimentos de assistência à saúde [1].

Os estudos de simulação de alta fidelidade e com recurso a paciente padrão na área da enfermagem comunitária ainda são incipientes [6,7]. Além disso, o investimento no desenvolvimento de medidas adequadas de avaliação dos resultados da aprendizagem ainda é insuficiente. Logo, desenvolver estudos com instrumentos já existentes com novas populações, amostras e locais contribui para a validade e confiabilidade das pesquisas neste fenômeno de investigação [3]. Um desses instrumentos é a escala de design da simulação. Esta tem por objetivo avaliar a estruturação dos cenários de simulação [1].

A investigação em diferentes contextos do ensino de enfermagem a partir do uso da simulação contribui para o desenvolvimento de evidências, expansão da aplicabilidade de seu uso e para a melhoria da qualidade da formação profissional. Nessa perspectiva, o estudo teve por objetivo identificar a percepção de estudantes de enfermagem sobre as dimensões estruturais do design de cenários de simulação de alta fidelidade na imunização de adultos no contexto da atenção primária à saúde.

\section{MÉTODOS}

Estudo descritivo, exploratório, quantitativo desenvolvido em uma universidade pública federal brasileira no período de maio a junho de 2017. O estudo foi submetido e aprovado junto ao Comitê de Ética em Pesquisa sob protocolo no 1.958 .827 .

A amostra inicial, do tipo não probabilística por conveniência, foi de 29 estudantes que atenderam aos seguintes critérios de inclusão: ser estudante da graduação em enfermagem, obter frequência superior igual ou superior a $75 \%$ de um curso de formação de 40 horas e participar nos três cenários de simulação. Foram excluídos os estudantes que obtiverem frequência inferior a $75 \%$; os que não estiverem presentes nas simulações e aplicação dos instrumentos da pesquisa; bolsistas e colaboradores que contribuíram para a execução do estudo. Após serem aplicados os critérios de inclusão, a amostra final foi constituída de 17 estudantes.

Após concluir as etapas de exposição dialogada e treino de habilidades, os estudantes participaram em três cenários de simulação. Os cenários de simulação foram construídos e executados previamente. Um ambiente de sala de vacina foi recriado no laboratório de enfermagem. Os três cenários foram executados em um único dia, total de 1 hora para cada cenário. Para a execução dos cenários, foi utilizada a ferramenta paciente-padrão [1].

Três atores foram treinados para atuarem como pacientes. Estes foram selecionados a partir de suas 
características e experiência em atuação em simulações. Após reunião com os pesquisadores, foram treinados quanto ao posicionamento na cena, falas, expressões e postura, e orientados a responder somente o que estava previsto no script do cenário. O treinamento ocorreu no momento de balizamento da coleta de dados e teve carga horária de 4 horas. Além disso, antes da execução dos cenários, foram testados quanto a desenvoltura e aptidão em falar e expressar as orientações e gestos previstas no script.

Ao término das simulações, o grupo de intervenção participou do momento de discussão e reflexão, sendo utilizada a técnica do debriefing. No momento, utilizou-se o debriefing estruturado em três etapas, a saber: descrição, análise e síntese. Cada sessão durou 30 minutos. O debriefing é uma etapa em que todos os estudantes podem discutir sobre a cena vivenciada. Nesse momento, os estudantes tiveram a oportunidade de explorar os cenários vivenciados, ajudando-os a consolidar as informações adquiridas, identificar e refletir sobre as áreas em que podem melhorar [8].

A Figura 1 apresenta o diagrama de seguimento da pesquisa, os objetivos dos cenários executados e a composição da amostra final na aplicação da The Simulation Design Scale (student version). Em consonância com os cenários, foram estabelecidos três objetivos de aprendizagem, a saber: no cenário 1 , o objetivo era que o estudante realizasse o manejo do ponto de vista da imunização - de um paciente acometido por um acidente traumático; no cenário 2 , objetivou-se que o estudante realizasse o aprazamento e a administração de imunobiológicos conforme necessidade do paciente; e no cenário 3 , o objetivo era que o estudante reconhecesse uma situação de contraindicação de imunobiológicos.

A Escala de Design da Simulação foi utilizada para avaliar a estruturação dos cenários de simulação. A escala foi desenvolvida em 2004 pela National League for Nursing, com a finalidade de avaliar a estrutura de cenários de simulação. No estudo, foi utilizada a versão da escala The Simulation Design Scale (student version) validada para a língua portuguesa [9]. É válido destacar que após o estudo de propriedades psicométricas da Escala de Design de Simulação, realizado entre 2007 e 2010 com 2.200 participantes, tem-se referências que esta escala tem confiabilidade e validade suficiente para ser utilizada na pesquisa educacional [10].

A escala é composta por cinco dimensões, a saber: objetivos e informações; apoio; resolução de problemas; feedback e reflexão; realismo. Estas dimensões têm contemplados 20 itens e tem ainda duas subescalas: concordância e importância.

Na subescala de concordância, encontram-se 5 itens de avaliação e uma opção de não aplicável à simulação desenvolvida: 1 - Discordo totalmente da afirmação; 2 - Discordo da afirmação; 3 - Indeciso - nem concordo nem discordo da afirmação; 4 - Concordo com a afirmação; 5 - Concordo totalmente com a afirmação; NA - Não aplicável, a declaração não diz respeito à atividade simulada realizada.

Já a subescala de importância, possui os seguintes itens: 1 - Não é importante; 2 - Um pouco importante; 3 - Neutro; 4 - Importante; 5 - Muito Importante. Os dados foram analisados pelo IBM SPSS Statistics ${ }^{\circledR}$ versão 24 .

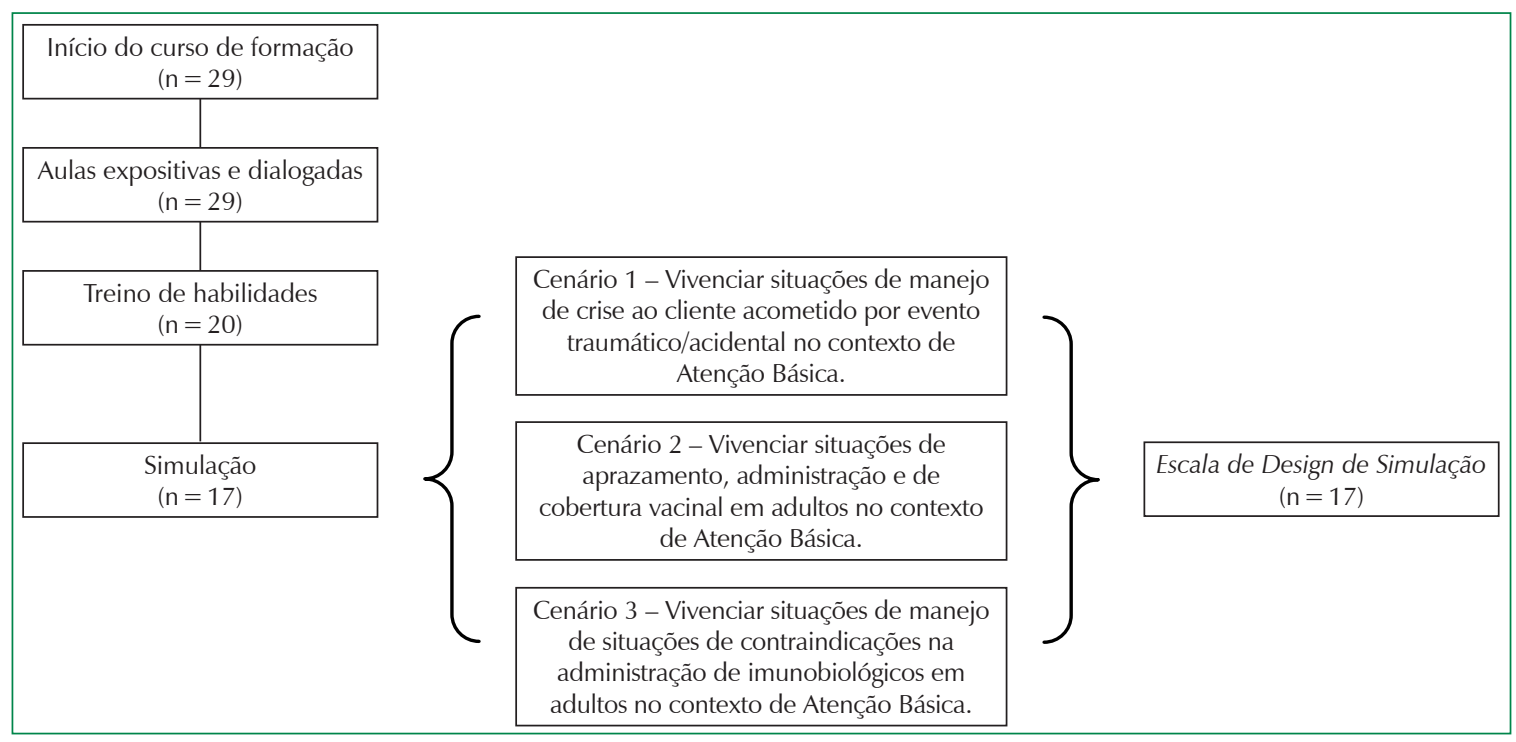

Figura 1. Diagrama de seguimento. Natal, 2017. 


\section{RESULTADOS}

A maioria dos estudantes eram do sexo feminino $(82,4 \%)$. A faixa etária mais frequente foi de 19 a 22 anos (64,7\%), média de 22,7, com idade mínima de 19 e máxima de 31 anos.

$\mathrm{Na}$ análise de consistência interna, a Escala de Design de Simulação apresentou um alpha de Cronbach total de 0,930 . Na subescala de concordância, o alpha foi de 0,931 . Já na subescala de importância, o alpha foi de 0,950. Em todas as dimensões da Escala de Design de Simulação, obteve-se médias igual ou superior a
4,60 e moda 5,00. Por apresentarem maior média e menor desvio padrão, as dimensões que apresentaram maior concordância e importância entres os estudantes foram, respectivamente, resolução de problemas e realismo.

Na Tabela 1, apresenta-se a pontuação global de concordância e importância nas cinco dimensões da escala de design de simulação. A Tabela 2 apresenta as médias, desvios padrão e moda das subescalas de concordância e importância. Nesta, são detalhados todo o conteúdo das cinco dimensões e suas respectivas representatividade estatística.

Tabela 1. Pontuação global de concordância e importância das dimensões da Escala de Design da Simulação (n=17) - Natal, 2017.

\begin{tabular}{|c|c|c|c|c|c|c|c|c|c|}
\hline Dimensões & Média & DP & Mediana & Moda & Max & Min & P25 & P50 & P75 \\
\hline \multicolumn{10}{|l|}{ Concordância } \\
\hline Objetivos e informações & 4,68 & 0,36 & 4,80 & 5,00 & 5,00 & 4,00 & 4,40 & 4,80 & 5,00 \\
\hline Apoio & 4,61 & 0,44 & 4,75 & 5,00 & 5,00 & 3,00 & 4,25 & 4,75 & 5,00 \\
\hline Resolução de problemas & 4,74 & 0,36 & 5,00 & 5,00 & 5,00 & 4,00 & 4,50 & 5,00 & 5,00 \\
\hline Feedback/Reflexão & 4,73 & 0,39 & 5,00 & 5,00 & 5,00 & 4,00 & 4,50 & 5,00 & 5,00 \\
\hline Realismo & 4,70 & 0,41 & 5,00 & 5,00 & 5,00 & 4,00 & 4,25 & 5,00 & 5,00 \\
\hline \multicolumn{10}{|l|}{ Importância } \\
\hline Objetivos e informações & 4,68 & 0,41 & 5,00 & 5,00 & 5,00 & 3,00 & 4,50 & 5,00 & 5,00 \\
\hline Apoio & 4,60 & 0,45 & 4,75 & 5,00 & 5,00 & 3,00 & 4,12 & 4,75 & 5,00 \\
\hline Resolução de problemas & 4,75 & 0,35 & 5,00 & 5,00 & 5,00 & 4,00 & 4,50 & 5,00 & 5,00 \\
\hline Feedback/Reflexão & 4,70 & 0,41 & 5,00 & 5,00 & 5,00 & 4,00 & 4,25 & 5,00 & 5,00 \\
\hline Realismo & 4,82 & 0,30 & 5,00 & 5,00 & 5,00 & 4,00 & 4,50 & 5,00 & 5,00 \\
\hline
\end{tabular}

Tabela 2. Concordância e importância das dimensões da Escala de Design da Simulação (n=17) - Natal, 2017.

\begin{tabular}{|c|c|c|c|c|c|c|}
\hline \multirow{2}{*}{ Variáveis/Dimensões } & \multicolumn{3}{|c|}{ Concordância } & \multicolumn{3}{|c|}{ Importância } \\
\hline & Média & DP & Moda* & Média & DP & Modat \\
\hline \multicolumn{7}{|l|}{ Objetivos e informações } \\
\hline 1. No início da simulação foi fornecida informação suficiente para proporcionar orientação e incentivo. & 4,88 & 0,33 & 5,00 & 4,82 & 0,39 & 5,00 \\
\hline 2. Eu entendi claramente a finalidade e os objetivos da simulação. & 4,76 & 0,43 & 5,00 & 4,64 & 0,49 & 5,00 \\
\hline 3. A simulação forneceu informação suficiente, de forma clara, para eu resolver a situação-problema. & 4,64 & 0,49 & 5,00 & 4,70 & 0,46 & 5,00 \\
\hline 4. Foi-me fornecida informação suficiente durante a simulação. & 4,64 & 0,49 & 5,00 & 4,64 & 0,49 & 5,00 \\
\hline 5. As pistas foram adequadas e direcionadas para promover a minha compreensão. & 4,47 & 0,62 & 5,00 & 4,58 & 0,61 & 5,00 \\
\hline \multicolumn{7}{|l|}{ Apoio } \\
\hline 6. O apoio foi oferecido em tempo oportuno. & 4,64 & 0,60 & 5,00 & 4,52 & 0,62 & 5,00 \\
\hline 7. A minha necessidade de ajuda foi reconhecida. & 4,58 & 0,50 & 5,00 & 4,64 & 0,49 & 5,00 \\
\hline 8. Eu senti-me apoiado pelo professor durante a simulação. & 4,58 & 0,50 & 5,00 & 4,70 & 0,46 & 5,00 \\
\hline 9. Eu fui apoiado no processo de aprendizagem. & 4,64 & 0,49 & 5,00 & 4,52 & 0,62 & 5,00 \\
\hline \multicolumn{7}{|l|}{ Resolução de problemas } \\
\hline 10. A resolução de problemas de forma autônoma foi facilitada. & 4,76 & 0,43 & 5,00 & 4,76 & 0,43 & 5,00 \\
\hline 11. Fui incentivado a explorar todas as possibilidades da simulação. & 4,82 & 0,39 & 5,00 & 4,70 & 0,46 & 5,00 \\
\hline 12. A simulação foi projetada para o meu nível específico de conhecimento e habilidades. & 4,70 & 0,46 & 5,00 & 4,76 & 0,43 & 5,00 \\
\hline 13. A simulação permitiu-me a oportunidade de priorizar as avaliações e os cuidados de enfermagem. & 4,70 & 0,58 & 5,00 & 4,82 & 0,39 & 5,00 \\
\hline 14. A simulação proporcionou-me uma oportunidade de estabelecer objetivos para o meu paciente. & 4,70 & 0,46 & 5,00 & 4,70 & 0,46 & 5,00 \\
\hline \multicolumn{7}{|l|}{ Feedback/Reflexão } \\
\hline 15. O feedback fornecido foi construtivo. & 4,70 & 0,46 & 5,00 & 4,76 & 0,43 & 5,00 \\
\hline 16. O feedback foi fornecido em tempo oportuno. & 4,82 & 0,39 & 5,00 & 4,82 & 0,39 & 5,00 \\
\hline 17. A simulação permitiu-me analisar meu próprio comportamento e ações. & 4,82 & 0,39 & 5,00 & 4,70 & 0,46 & 5,00 \\
\hline $\begin{array}{l}\text { 18. Após a simulação houve oportunidade para obter orientação /feedback do professor, a fim de } \\
\text { construir conhecimento para outro nível. }\end{array}$ & 4,58 & 0,61 & 5,00 & 4,52 & 0,62 & 5,00 \\
\hline \multicolumn{7}{|l|}{ Realismo } \\
\hline 19. O cenário se assemelhava a uma situação da vida real. & 4,52 & 0,62 & 5,00 & 4,76 & 0,43 & 5,00 \\
\hline 20. Fatores, situações e variáveis da vida real foram incorporados ao cenário de simulação. & 4,76 & 0,43 & 5,00 & 4,88 & 0,33 & 5,00 \\
\hline
\end{tabular}

Fonte: Originário da própria pesquisa.

* Concordância: 1 - Discordo totalmente da afirmação; 2 - Discordo da afirmação; 3 - Indeciso - nem concordo nem discordo da afirmação; 4 - Concordo com a afirmação; 5 - Concordo totalmente com a afirmação; NA - Não aplicável, a declaração não diz respeito à atividade simulada realizada.

†Importância: 1 - Não é importante; 2 - Um pouco importante; 3 - Neutro; 4 - Importante; 5 - Muito Importante. 
Como se pode observar, na dimensão de objetivos e informações a média global foi de 4,68. Destaca-se o item 1 , por apresentar maior média $(4,88)$ e menor desvio padrão $(0,33)$ de concordância e importância. No que se refere ao apoio, as médias globais também foram bastante aproximadas (4,60 para concordância e 4,61 para importância).

Quanto à dimensão da resolução de problemas, destaca-se a forte concordância dos estudantes em relação ao incentivo que lhes foram dados para a exploração das possibilidades da simulação (média $=4,82$ e DP $= \pm 0,39$ ). Ao mesmo, os estudantes sinalizaram a importância da oportunidade de priorizar as avaliações e os cuidados de enfermagem durante a simulação (média $=4,82$ e DP $= \pm 0,39$ ).

O feedback/reflexão também foi uma dimensão avaliada positivamente pelos estudantes. As médias globais foram de 4,73 para concordância e 4,70 para importância.

Na dimensão que se detém ao realismo, as médias globais de concordância e importância foram de 4,70 e 4,82 respectivamente. Os estudantes concordam que fatores e variáveis da vida real foram incorporados aos cenários simulados. Entretanto, as médias de importância (itens 19 e 20 da escala) foram superiores às de concordância.

\section{DISCUSSÃO}

O estudo identificou a percepção de estudantes de enfermagem sobre as dimensões estruturais do design de cenários de simulação de alta fidelidade. A partir da participação em três cenários de simulação aplicados a situações de imunização de adultos no contexto da atenção primária à saúde, os estudantes avaliaram positivamente as cinco dimensões da escala de design de simulação. De modo semelhante, um estudo com 66 estudantes de enfermagem que utilizou a Escala de Design de Simulação em três cenários de pediatria, mostrou que o apoio (média $=4,41$ ), feedback/reflexão $($ média $=4,47)$ e o realismo $($ média $=4,50)$ foram os domínios mais reportados como relevantes pelos participantes do estudo [11].

No que se refere aos objetivos e informações, ressalta-se que todas as experiências baseadas em simulação devem começar com o desenvolvimento de objetivos mensuráveis projetados para alcançar os resultados esperados. Os objetivos são as ferramentas que orientam e facilitam a realização da simulação, podem ser gerais ou específicos [12]. Neste estudo, os estudantes concordam e consideram relevante que a simulação disponha de objetivos claros e bem definidos para orientar as ações durante a execução dos cenários.

Quanto à definição dos objetivos, a taxonomia de Bloom orienta e fornece a estrutura para desenvolver e nivelar os objetivos para os resultados da aprendizagem esperados. Os objetivos podem ser de natureza afetiva, cognitiva e psicomotora [13]. É importante destacar que cada domínio apresenta níveis hierárquicos. Além disso, salienta-se que se avalie se cada nível hierárquico foi atingido pelos objetivos antes de passar a um nível superior [12].

A observância destes níveis hierárquicos facilita a resolução dos problemas encontrados pelos estudantes a partir da experiência baseada em simulação. É de fundamental importância que o planejamento dos cenários leve em consideração o nível específico de conhecimento e habilidades dos estudantes. Com isso, os estudantes têm a capacidade de estabelecer, priorizar e avaliar as suas ações e condutas frente aos problemas identificados nos casos simulados.

Para a criação de cenários de simulação, existe uma orientação para a construção dos objetivos: a estrutura S.M.A.R.T (Specific; Measurable; Achievable; Realistic; Time phased). Esta estrutura leva em consideração o objetivo específico, a forma de mensuração dos resultados a partir do objetivo, se este é alcançável e como alcança-lo, se é realista e o tempo necessário para alcança-lo. Não seguir este padrão pode levar a consequências como resultados indesejados ou não previstos e diminuição da satisfação dos participantes [12].

Além disso, no início da simulação, é importante que o facilitador informe os participantes sobre as diversas interfaces do cenário: ambientação do cenário, esclarecimento dos objetivos, descrição do caso, manipulação prévia de equipamentos disponíveis no cenário, possibilidades de ajuda e ou intervenção de outros sujeitos durante a execução do cenário e entre outros aspectos.

$\mathrm{O}$ apoio e o esclarecimentos sobre os objetivos a serem alcançados durante os cenários, são elementos fundamentais para a preparação do estudante na vivência da simulação e pode contribuir para a sua aprendizagem [11]. Na pesquisa, a maioria dos estudantes sentiram-se apoiados durante o processo de aprendizagem e reconhecem a importância do apoio nos cenários simulados.

O briefing é uma sessão de informação ou orientação realizada antes do início de uma simulação em que instruções ou informações preparatórias são dadas aos participantes. Estas informações objetivam oferecer um ambiente seguro e auxiliar os participantes 
na realização dos objetivos de simulação [12]. O briefing é um elemento crítico para os participantes alcançarem com sucesso os objetivos na simulação. Além disso, prepara o estudante para melhor refletir sobre o seu desempenho, reduz a ansiedade destes relacionadas a simulação, melhora as competências e a autoconfiança. Logo, é tão importante quanto o debriefing [14]. A ausência ou um breve briefing, pode gerar comprometer a interação e desempenho dos estudantes [15].

Um estudo com 207 estudantes de enfermagem evidenciou que após passarem por etapas de briefing incluindo orientação oral, experiência prévia do ambiente de simulação e a prática de habilidades de enfermagem em laboratório obtiveram scores de competência clínica, de satisfação e de autoconfiança superiores ao grupo que não receberam essa intervenção [16]. Logo, as atividades de briefing podem ser desenvolvidas e integradas nas simulações, e consequentemente maximizar os resultados de aprendizagem dos estudantes [16].

$\mathrm{Na}$ percepção dos estudantes, o feedback foi construtivo e fornecido em tempo oportuno, permitiu analisar o comportamento e ações, e oportuno para obter informações do professor. Esses resultados estão em consonância com o que é orientado na literatura. $\mathrm{Na}$ simulação o feedback deve ser construtivo, fornecido em tempo oportuno e contar com a orientação do facilitador com o objetivo de construir conhecimento. O feedback e a reflexão são determinantes essenciais para o desenvolvimento de competências profissionais [17].

Portanto, os docentes de enfermagem precisam compreender o quão impactante podem ser os seus comentários durante o feedback. Muitas vezes, os educadores subestimam o impacto do feedback e a sua influência para a autoimagem do estudante [11].

Toda a experiência de simulação inclui uma sessão estruturada de debriefing com o objetivo de melhorar o desempenho dos participantes [12]. Há evidências que a aprendizagem ocorre na fase de debriefing durante a experiência baseada na simulação [12]. Logo, esta etapa deve ser facilitada por uma pessoa competente nesta metodologia, em ambiente que seja seguro, que promova a confidencialidade, a confiança, a comunicação aberta, a autoanálise, feedback e reflexão. Além disso, deve levar em consideração os objetivos e resultados traçados durante o planejamento da simulação [12].

As experiências baseadas em simulação objetivam avaliar o conhecimento, as habilidades, as atitudes, e o comportamento contidos nos domínios cognitivo, afetivo e psicomotor de aprendizagem [12]. Há diferentes formas de avaliar os resultados e o impacto de experiências baseadas em simulação como a satisfação dos participantes, aprendizagem/conhecimento, habilidades e atitudes adquiridas a partir da simulação, mudança de medidas de comportamento após o treinamento, e melhoria da qualidade e segurança, e aumento do retorno de investimento após o treinamento como produtividade e retenção dos funcionários [18].

Há três formas principais de avaliação que podem ser utilizadas a partir da simulação: somativa, formativa e a avaliação de alto risco. Para avaliar os participantes é indispensável que o facilitador determine a intenção da experiência baseada na simulação e faça uso de ferramentas válidas, confiáveis e que estas sejam utilizadas por avaliadores aptos a manipulá-las [12]. $\mathrm{Na}$ avaliação somativa pode-se utilizar um formato padronizado e métodos de pontuação. Em algumas situações, a avaliação de vários experts pode ser necessária. Recursos como vídeos podem ajudar nesse processo de atribuição de pontuação. Além disso, o estudante deve receber ofeedback das suas ações [12]. $\mathrm{Na}$ avaliação formativa, deve-se monitorar o progresso para alcançar os resultados, fornecer um feedback formativo contínuo, identificar e fechar lacunas de conhecimento e habilidade, e avaliar a prontidão para as experiências do mundo real. É importante que o avaliador seja responsável por um grupo de poucos estudantes. Já a avaliação de alto risco pode ser utilizada para identificar problemas de segurança significativos durante o processo de aprendizagem [12]. É importante destacar que a forma de avaliação vai ao encontro dos objetivos da aprendizagem. Ao planejar uma sessão de simulação é indispensável que o docente esteja qualificado e tenha em mente como avaliá-la.

Portanto, a eficácia dos resultados da aprendizagem desejados nas simulações pode ser influenciada pelo despreparo do docente que facilita as simulações. Logo, quando se pretende usar simulações no currículo, as instituições formadoras necessitam capacitar o corpo docente [11].

Além disso, é importante considerar que os resultados esperados devem estar de acordo com a organização e a missão do programa onde está vinculada a proposta da simulação, o currículo, as características dos participantes, avaliação das necessidades e área de interesse [12].

É fundamental configurar o ambiente de simulação como o mais próximo possível da configuração clínica real e permitir aos alunos experimentá-lo com antecedência [16]. No que diz respeito a fidelidade 
e realismo, os estudantes de enfermagem compreendem que quanto mais real a simulação, melhor é a aprendizagem [11]. Neste estudo, os estudantes atribuíram altos scores de importância sobre estes aspectos.

$\mathrm{Na}$ percepção dos estudantes, os cenários simulados foram claros e objetivos em relação à proposta e execução. Além disso, sentiram-se apoiados durante o processo de aprendizagem, o que permitiu explorar e resolver os problemas apresentados durante a simulação. O feedback fornecido foi relevante e oportunizou a reflexão dos estudantes quanto às suas práticas e posturas. Por fim, o realismo foi destacado como um aspecto importante pelos pesquisados. As dimensões de resolução de problemas e o realismo apresentaram maior concordância e importância na percepção dos pesquisados.

A Escala de Design de Simulação utilizada apresentou uma boa confiabilidade (alpha de Cronbach total de 0,930). Nas subescalas de concordância e importância, e nos vinte itens contidos nas dimensões apresentadas, as médias foram superiores a 4,50.

Uma limitação do estudo foi a amostra $(n=17)$. Além disso, por escassez na literatura, não foi possível comparar os resultados apresentados com outros estudos. Mais investigações utilizando testes psicométricos na área da simulação aplicadas ao ensino de enfermagem precisam ser desenvolvidos.

Espera-se que os resultados deste estudo contribuam para a difusão da relevância da utilização de instrumentos confiáveis que permitam avaliar e aperfeiçoar o planejamento e o design da simulação de alta fidelidade no contexto do ensino de enfermagem.

\section{NOTAS}

Declaração de conflito de interesses

Os autores declaram não haver conflitos de interesses relevantes ao conteúdo deste estudo.

Contribuições dos autores

Todos os autores fizeram contribuições substanciais para concepção, ou delineamento, ou aquisição, ou análise ou interpretação de dados; e redação do trabalho ou revisão crítica; e aprovação final da versão para publicação.

Disponibilidade dos dados e responsabilidade pelos resultados

Todos os autores declaram ter tido total acesso aos dados obtidos e assumem completa responsabilidade pela integridade destes resultados.

\section{REFERÊNCIAS}

1. National League for Nursing. Simulation Innovation Resource Center, 2013. Available from: http://sirc.nln.org/mod/ glossary/view.php?id=183

2. Foronda C, Liu S, Bauman EB. Evaluation of simulation in undergraduate nurse education: An integrative review. Clin Simul Nurs. 2013 [cited 2018 April 11]; 9(10):409-16.

3. Adamson KA, Kardong-Edgren S, Willhaus J. An updated review of published simulation evaluation instruments. Clin Simul Nurs. 2013 [cited 2018 April 11];9(9):393-400. Available from: http://dx.doi.org/10.1016/j. ecns.2012.09.004

4. Doolen J, Mariani B, Atz T, et al. High-Fidelity Simulation in Undergraduate Nursing Education: A Review of Simulation Reviews. Clin Simul Nurs. 2016 [cited 2018 April 11]; 12(7):290-302. https://doi.org/10.1016/j. ecns.2016.01.009

5. Durham CF, Cato ML, Lasater K. NLN/Jeffries Simulation Framework state of the science project: Participant construct. Clin Simul Nurs. 2014 [cited 2018 April 11]; 10(7):363-72. https://doi.org/10.1016/j.ecns.2014.04.002

6. Husson NM, Zulkosky K, Fetter M, Kamerer J. Integrating community health simulation scenarios: Experiences from the NCSBN National simulation study. Clinical Simulation in Nursing. 2014 [cited 2018 April 11]; 10:581-6. Available from: http://dx.doi.org/10.1016/j.ecns.2013.11.009

7. Herron EK, Nemeth J, Powers KA. Community Health Simulation with a Standardized Patient: Exploring the Experience. Clin Simul Nurs. 2017 [cited 2018 April 11]; 13(7): 331-7. Available from: http://dx.doi.org/10.1016/j. ecns.2017.05.011

8. Arthur C, Levett-Jones T, Kable A. Quality indicators for the design and implementation of simulation experiences: A Delphi study. Nurse Education Today. 2013 [cited 2018 April 11]; 33:1357-61. https://doi.org/10.1016/j. nedt.2012.07.012 
9. Almeida RGS, Mazzo A, Martins JCA. Validation to Portuguese of the Scale of Student Satisfaction and SelfConfidence in Learning. Rev Lat Am Enfermagem. 2015 [cited 2018 April 11]; 23(6):1007-13. Available from: http://www.scielo.br/scielo.php?script=sci_arttext\&pid=S010411692015000601007\&lng=en\&tlng=en. https://doi, org/10.1590/0104-1169.0472.2643

10. Franklin AE, Burns P, Lee CS. Psychometric testing on the NLN Student Satisfaction and Self-Confidence in Learning, Simulation Design Scale, and Educational Practices Questionnaire using a sample of pre-licensure novice nurses. Nurse Educ Today. 2014 [cited 2018 April 11]; 34(10):1298-304. https://doi.org/10.1016/j.nedt.2014.06.011

11. Cantrell MA, Meakim C, Cash K. Development and Evaluation of Three Pediatric-based Clinical Simulation. Clin Simul Nurs. 2008 [cited 2018 April 11]; 4(1):21-8. Available from: http://dx.doi.org/10.1016/j.ecns.2009.05.052

12. Committee IS. INACSL Standards of Best Practice: Simulation ${ }^{\text {SM }}$ Simulation Design. Clin Simul Nurs. 2016 [cited 2018 April 11]; 12:13-5. https://doi.org/10.1016/j.ecns.2016.09.005

13. Bloom BS. Taxonomy of educational objectives: The classification of educational goals. Handbook 1: Cognitive domain. New York: Longman; 1956.

14. Mcdermott DS. The prebriefing concept: a Delphi study of CHSE experts. Clinical Simulation in Nursing. 2016 [cited 2018 April 11]; 12(6):219-27. https://doi.org/10.1016/j.ecns.2016.02.001

15. Park SN, Chu MS, Hwang YY, et al. Effects of integrated nursing practice simulation-based training on stress, interest in learning, and problem-solving ability of nursing students. Journal of the Korean Academy of Fundamentals of Nursing. 2015 [cited 2018 April 11]; 22(4):424-32. Available from: http://dx.doi.org/10.7739/jkafn.2015.22.4.424

16. Kim YJ, Noh GO, Im YS. Effect of Step-Based Prebriefing Activities on Flow and Clinical Competency of Nursing Students in Simulation-Based Education. Clin Simul Nurs. 2017 [cited 2018 April 12]; 13(11):544-51. https://doi. org/10.1016/j.ecns.2017.06.005

17. Babenko-Mould Y, Andrusyszyn A, Goldenberg D. Effects of computer-based clinical conferencing on nursing students' self-efficacy. Journal of Nursing Education. 2004 [cited 2018 April 12]; 43(4):149-155.

18. Kirkpatrick DL. Evaluating training programs: The four levels. San Francisco: Berrett-Koehler Publishers; 1994. https://doi.org/10.1016/s1098-2140(99)80206-9 